\title{
ESTRATÉGIAS DE APRENDIZAGEM UTILIZADAS POR ALUNOS DO CURSO DE PEDAGOGIA DE UMA IES PÚBLICA DO ESTADO DO PARANÁ OFERTADO A DISTÂNCIA
}

\author{
Natália Moraes Góes - UEL \\ nataliamoraesg@gmail.com \\ Marilza Aparecida Pavesi - UEL \\ maripavesi@gmail.com \\ Paula Mariza Zedu Alliprandini - UEL \\ paulaalliprandini@uel.br
}

\begin{abstract}
Resumo
O objetivo deste trabalho foi verificar o uso de estratégias de aprendizagem utilizadas por alunos de um curso de Pedagogia ofertado a distância de uma IES pública do Estado do Paraná. Participaram da pesquisa 1089 alunos de um curso de Pedagogia, sendo 1076 do sexo feminino e 13, do masculino, com idade entre 25 a 69 anos. Para a coleta de dados foi disponibilizada na plataforma do curso a "Escala de Estratégias de Aprendizagem (EEA)" validada por Zerbini, Carvalho e Abbad (2008), composta pelos fatores: controle da emoção, busca de ajuda interpessoal, repetição e organização, controle da motivação, elaboração, busca de ajuda no material didática e monitoramento da compreensão. Por meio da análise de variância, os resultados evidenciaram diferenças significativas entre os fatores de análise propostos pela escala, sendo o monitoramento da compreensão e controle da emoção, os fatores que apresentaram menor frequência. Os resultados trazem importantes implicações educacionais para o processo ensino-aprendizagem na $\mathrm{EaD}$.
\end{abstract}

Palavras-chave: Estratégias de aprendizagem, educação a distância $(\mathrm{EaD})$, processamento da informação, pedagogia.

\section{Learning Strategies Used by Students from the Course of Pedagogy as Distance Learning Offered of a Public College in the State of Paraná}

\begin{abstract}
The goal of this work was to verify the use of learning strategies used by students of an Education course offered as distance learning from a public College in the State of Paraná, Brazil. In the study 1089 students of a pedagogy course took part, being 1076 females and 13 males, with ages between 25 and 69 years. For data collection it was made available in the platform of the course the "Scale of Learning Strategies (SLS)" validated by Zerbini, Carvalho and Abbad (2008), composed by factors: emotion control, searching for interpersonal help, repetition and organization, motivation control, elaboration, searching for help in the didactic materials and monitoring of comprehension. By a variance analysis, the results evidenced significant differences between the analysis factors proposed by the scale, being comprehension monitoring and emotional control the factors that had lower frequencies. The results bring important educational implications for the teaching-learning process in Distance Learning.
\end{abstract}

Key-words: Learning strategies, distance learning, information processing, pedagogy. 
Introdução

A Educação a Distância (EaD) no Brasil é uma modalidade de ensino em franca expansão, alcançando todas as esferas educacionais do país. De acordo com Tannous e Ropoli (2005), a EaD tem se tornado uma excelente opção para o desenvolvimento e implantação das mais diversas propostas pedagógicas, que vão desde o método assíncrono, no qual é priorizada a instrução programada, sem nenhuma interação, até propostas educacionais inovadoras, caracterizadas pela interação entre os participantes e o desenvolvimento do trabalho colaborativo.

$\mathrm{O}$ avanço da tecnologia com a disseminação das tecnologias de informação e comunicação (TICs) tornou-se um grande aliado no cenário da formação educacional. Por meio de cursos a distância mediados por computador é possível atender tanto aos interesses dos estudantes na busca de novas aprendizagens, como aos interesses institucionais.

Segundo a Associação Brasileira de Educação a Distância (ABED, 2012), em 2012 haviam 3.971 cursos autorizados pelo MEC e neles estudavam em torno de 800.000 alunos, sendo que a maior parte deles estavam matriculados no ensino superior (75\%). A pós-graduação, em todos os níveis, respondia por $17,5 \%$ dos estudantes e o restante dos alunos $(7,5 \%)$ se dividiam entre cursos de ensino fundamental, médio e técnico. Entre os estudantes dos cursos reconhecidos pelo MEC, 53\% estavam na região sul do país.

A EaD, em seu projeto inicial, foi apresentada como uma possibilidade de democratização do ensino (BOHADANA; VALLE, 2009). Um dos motivos para essa caracterização se vale pelo seu caráter mais flexível quando comparado a outras modalidades de ensino mais tradicionais.

Se por um lado, a EaD pode ser considerada uma forma mais flexível, por outro, esta característica requer diferentes atitudes e comportamentos dos agentes que dela fazem parte. Entre os alunos, espera-se que possam agir independentemente, refletir sobre a própria aprendizagem e controlá-la (FARIA, 2010), ou seja, requer indivíduos autônomos, competentes e críticos. Nesse sentido, o uso das estratégias de aprendizagem torna-se uma excelente ferramenta para que eles conheçam e regulem sua própria cognição.

Coll e Monereo (2010) afirmam que a Psicologia da Educação, como área que estuda as mudanças psicológicas que ocorrem nas pessoas, como consequência de sua participação em situações e atividades educacionais, deve colocar em local privilegiado o estudo das mudanças provocadas pelo uso total ou parcial das TICs. Frente a essa realidade, a partir da Teoria do Processamento da Informação, proposta pelos teóricos cognitivistas, pesquisadores têm investigado sobre o uso de estratégias de aprendizagem, apostando na diminuição das dificuldades de aprendizagem, quando essas ferramentas são ensinadas e adequadas às variadas situações que o indivíduo pode se deparar (BORUCHOVITCH, 1999; COSTA; BUROCHOVITCH, 2009; LINS; ARAUJO; MINERVINO, 2011; BUSNELLO; JOU; SPERB, 2012). Entretanto, esses estudos restringem-se ao ensino presencial. Quando a busca se realiza com enfoque na $\mathrm{EaD}$, são poucos os trabalhos encontrados que se preocupam com essa temática (ZERBINI; ABBAD, 2005, 2008; ABBAD; CORREAA; MENESES, 2010; ABBAD et al., 2012).

De acordo com Warr e Allan (1998, apud ZERBINI; PILATI, 2012, p. 228): 
treino, utilizadas pelos indivíduos em atividade de aprendizagem que facilitam o alcance de todos os critérios da aprendizagem.

Joly (2004) propõe que as estratégias de aprendizagem sejam divididas entre estratégias cognitivas e estratégias metacognitivas. As estratégias cognitivas são aquelas utilizadas especialmente para ajudar o aprendiz a assimilar as informações quando estão frente a uma tarefa, refere-se a comportamentos e pensamentos que influenciam o processo de aprendizagem, a fim de que as informações possam ser armazenadas mais eficientemente. Dentre as estratégias cognitivas, pode se apontar as estratégias de ensaio, elaboração e organização. As estratégias metacognitivas referem-se simultaneamente, ao estabelecimento de objetivos de estudo, o conhecimento sobre a própria compreensão e o conhecimento de como compreender. As estratégias metacognitivas estão relacionadas à autorregulação dos processos cognitivos. Por estratégias metacognitivas, reconhecem-se as estratégias de planejamento, monitoramento e regulação (BORUCHOVITCH, 1999).

No contexto escolar, espera-se que os aprendizes disponham de uma variedade de estratégias de aprendizagem para a melhor condução da sua aprendizagem. Neste sentido, Costa e Boruchovitch (2000 apud LEME, 2010, p. 25) ressaltam: "[...] aluno pode ter conhecimento sobre si, sobre a tarefa e conhecer diferentes estratégias, porém, se não souber como se apropriar, monitorar e regular seu aprendizado, poderá não ter um bom desempenho". Diante disso, ressalta-se a necessidade das estratégias cognitivas e metacognitivas acontecerem de forma interligada, para que atinjam a aprendizagem. Entretanto, mesmo interligadas, cada uma possui suas próprias características.

Pensando no que foi exposto acima e relacionando com o contexto escolar, à guisa do referencial teórico da Teoria do Processamento da Informação, o uso de estratégias se torna essencialmente significante neste contexto, principalmente aquelas que desenvolvem as capacidades metacognitivas, pois são consideradas nesta teoria, o elemento central para a aprendizagem (CORSO et al., 2013). Com vista a indivíduos mais autônomos, capazes de pensar sobre sua própria cognição e autônomos com relação à sua aprendizagem, o ensino e o uso de estratégias de aprendizagem torna-se fundamental no ensino regular, como também em outros espaços formais de educação, como na EaD.

Diante da realidade brasileira, onde a EaD tem sido cada vez mais disseminada, ressalta-se a importância de investigar esta modalidade de ensino. Nesse sentido, a presente pesquisa, embasada na perspectiva teórica da Psicologia Cognitiva/Teoria do Processamento da Informação, tem como objetivo verificar o uso das estratégias de aprendizagem por alunos de um curso de Pedagogia a distância ofertado por uma IES pública do Estado do Paraná.

\section{Material e Métodos}

\section{Participantes}

Participaram da presente pesquisa 1089 alunos do curso de Pedagogia a distância ofertado por uma IES pública do estado do Paraná. Do total de participantes, 1076 eram do sexo feminino e 13 do sexo masculino. A faixa etária dos participantes variou de 25 a 69 anos de idade. 


\section{Instrumento de Coleta de Dados}

A escala utilizada para este estudo foi desenvolvida e validada por Zerbini, Carvalho e Abbad (2008, apud ZERBINI; PILATI, 2012), intitulada "Escala de Estratégias de Aprendizagem (EEA)".

A escala caracteriza-se como do tipo Likert, tendo como opção de respostas de 0 (nunca) a 10 (sempre). A escala é composta por 28 itens, dividida em 7 fatores, apresentados a seguir: 1. controle da emoção: são as estratégias autorregulatórias de controle de ansiedade e controle da concentração, itens 1 a 5 do instrumento; 2 . busca de ajuda interpessoal: estratégias comportamentais de obtenção de auxílio de outras pessoas para tirar dúvidas, itens 6 a 11 da escala; 3. repetição e organização: são as estratégias cognitivas de repetição do material para posteriormente realizar esquemas mentais do material estudado, itens 12 a 16; 4. controle da motivação: estratégias de autorregulação de controle da motivação e da atenção, os itens 17 a 20; 5. elaboração: estratégias cognitivas de reflexão sobre implicações e conexões entre o material aprendido e o conhecimento existente do aluno, os itens 21 a $23 ; 6$. busca de ajuda no material didático: estratégias comportamentais de obtenção de informação no material do curso ou em outras fontes, excluem-se as que envolvem contato social, itens 24 a 25 e 7. monitoramento da compreensão: estratégias autorregulatórias de avaliação do próprio processo de aprendizagem e modificação do comportamento, os itens (26 a 28) (ZERBINI; PILATI, 2012).

De acordo com Zerbini e Pilati (2012) seria considerado baixo uso de estratégias de aprendizagem, valores médios entre 0 e 4; 4,1 a 7 indicam uso moderado das estratégias e entre 7,1 a 10, uso frequente das estratégias ao longo do curso.

\section{Procedimentos}

Inicialmente, o presente projeto tramitou e foi aprovado pelo Comitê de Ética da IES pesquisada, conforme parecer 071/2013. Para a coleta de dados, foram utilizados os recursos da ferramenta Google Drive. O link da pesquisa foi disponibilizado na plataforma do referido curso e, por meio desta ferramenta, o aluno podia acessar e responder a Escala online e as respostas automaticamente eram disponibilizadas por meio da emissão de um relatório.

Ao acessar o link, o aluno se deparava com uma página que apresentava o Termo de Consentimento Livre e Esclarecido (TCLE). Após a leitura do termo e assinalar a concordância em participar da pesquisa, abria-se uma nova página onde a Escala de Estratégias de Aprendizagem estava disponível para ser respondida. O link da pesquisa ficou disponível na plataforma nos meses de Junho e Julho de 2013. Após este período, o link foi retirado da plataforma e posteriormente foram realizadas as análises dos dados, por meio do software SPSS.

\section{Resultados e Discussões}

Com base nos resultados obtidos a partir da aplicação da Escala de Estratégias de Aprendizagem (EAA) foram calculados os valores mínimos e máximos, as médias e o desvio padrão, para cada Fator da Escala obtido a partir das estimativas dos participantes. A Tabela 1 indica os valores encontrados. 
Tabela 1 - Índice dos valores mínimos, máximos, médias e desvio padrão, referente a

\begin{tabular}{lcccc}
\hline \multirow{2}{*}{ Fatores } & \multicolumn{4}{c}{ Valores N=1089 } \\
\cline { 2 - 5 } & Mínimo & Máximo & Médias & DP \\
\hline 1. Controle da Emoção & 0 & 10 & 7,46 & 1,82 \\
2. Busca de ajuda interpessoal & 0 & 10 & 8,10 & 1,69 \\
3. Repetição e organização & 0 & 10 & 8,97 & 1,17 \\
4. Controle da Motivação & 0 & 10 & 8,90 & 1,28 \\
5. Elaboração & 0 & 10 & 9,42 & 0,96 \\
6. Busca de ajuda ao material didático & 0 & 10 & 8,99 & 1,44 \\
7. Monitoramento da compreensão & 0 & 10 & 8,05 & 2,00 \\
\hline
\end{tabular}

Legenda: DP: desvio padrão

cada fator da Escala de Estratégias de Aprendizagem (EAA).

Fonte: Autoras (2013).

Conforme apresentado na Tabela 1 , os resultados indicam que, independente do Fator investigado, os valores mínimos e máximos variaram entre 0 (zero) e 10 (dez). Sobre as médias encontradas, pode-se considerar que os participantes desta pesquisa fazem uso frequente de estratégias de aprendizagem, visto que as médias variaram de 7,46 a 9,42 .

Pesquisa anterior de Abbad, Corrêa e Meneses (2010) registraram médias inferiores às encontradas no presente estudo. As médias encontradas pelos autores variaram de 3,72 a 7,76, resultado este que indicou pouca e moderada frequência no uso das estratégias de aprendizagem. Borges e Junior (2012) também buscaram investigar o uso de estratégias de aprendizagem entre alunos de curso a distância e encontraram resultados divergentes do encontrado no presente estudo. Os alunos foram classificados em nível médio, quanto ao uso de estratégias de aprendizagem. Entretanto, os autores reconhecem que mesmo que os participantes não tenham sido classificados em nível alto no uso das estratégias, o uso das mesmas parece apropriado às necessidades de aprendizagem em EaD (BORGES; JUNIOR; 2012).

Diante dos dados obtidos, mesmo com baixa variação das médias entre os fatores, destaca-se que o Fator 5 apresentou uma maior média quando comparado às demais médias. O Fator 5, definido como de Elaboração, refere-se às estratégias cognitivas de reflexão sobre o material aprendido e o conhecimento já existente. Este resultado converge com o encontrado anteriormente por Zerbini e Abbad (2008), cujas autoras também apontaram este fator como o que apresentou maior frequência quando comparado a outros Fatores.

Abbad, Corrêa e Meneses (2010) afirmam que curso a distância requer atitudes e comportamentos diferenciados, por exemplo: que os alunos tenham uma postura autônoma, frente ao ato de estudar e que desenvolvam características distintas para o acompanhamento do curso a distância em que estão inseridos, pelo caráter distinto que os cursos de $\mathrm{EaD}$ têm quando comparado ao ensino regular. Entre estes comportamentos destaca-se também a autoaprendizagem, elemento crucial para $o$ sucesso dos alunos na $\mathrm{EaD}$, isto é "a eficácia depende em grande parte da motivação do estudante e de suas condições de estudo" (KEEGAN apud BELLONI, 2008, p.30).

Comportamentos como estes, indicados por Abbad, Corrêa e Meneses (2010) e Keegan (1983 apud BELLONI, 2008) relacionados sobre as expectativas em relação aos 
alunos da $\mathrm{EaD}$, foram evidenciados nesta pesquisa. Assim, quando questionados sobre a busca em outras fontes que não envolvam contato social, Fator 6 , os alunos indicaram média alta com relação a esse comportamento $(8,99)$. Este resultado revela que os alunos frente à autonomia que o curso proporciona, têm buscado diferentes formas de obtenção de informações, não se satisfazendo apenas com as informações trazidas pelo professor ou pelo tutor, mas sim, têm buscado ir além, desenvolvendo atitudes de autonomia e autoaprendizagem. Estudo realizado por Borges e Junior (2012) converge com o resultado encontrado neste estudo, quando afirmam "[...] percebemos uma aparente tendência desses alunos a darem os próprios passos em direção ao aprendizado" (BORGES; JUNIOR, 2012, p.70).

$\mathrm{Na}$ sequência, os dados foram submetidos à análise de variância aplicada aos escores obtidos para os sete fatores, tendo sido evidenciada diferenças significativas entre os fatores da escala $\left[F_{(7616,6)}=226,29, p \leq 0,05\right]$. Por meio do teste de Tukey, foram evidenciadas diferenças significativas entre alguns fatores, as quais podem ser visualizadas na Tabela 2, apresentada a seguir.

Tabela 2 - Comparação e busca de diferenças estatisticamente significativas entre os Fatores.

\begin{tabular}{|c|c|c|c|c|c|c|c|c|c|c|c|c|c|}
\hline \multicolumn{14}{|c|}{ Comparações entre Fatores } \\
\hline \multicolumn{2}{|c|}{$\begin{array}{c}1 \\
\text { Controle da } \\
\text { emoção }\end{array}$} & \multicolumn{2}{|c|}{$\begin{array}{c}2 \\
\text { Busca de } \\
\text { ajuda } \\
\text { interpessoal }\end{array}$} & \multicolumn{2}{|c|}{$\begin{array}{c}3 \\
\text { Repetição e } \\
\text { organização }\end{array}$} & \multicolumn{2}{|c|}{$\begin{array}{c}4 \\
\text { Controle } \\
\text { da } \\
\text { motivação }\end{array}$} & \multicolumn{2}{|c|}{$\begin{array}{c}5 \\
\text { Elaboração }\end{array}$} & \multicolumn{2}{|c|}{$\begin{array}{c}6 \\
\text { Busca de } \\
\text { ajuda no } \\
\text { material } \\
\text { didático } \\
\end{array}$} & \multicolumn{2}{|c|}{$\begin{array}{c}7 \\
\text { Monitoramento } \\
\text { da } \\
\text { compreensão }\end{array}$} \\
\hline 2 & $-0,65^{*}$ & 1 & 0,65 & 1 & $1,51^{*}$ & 1 & $1,44^{*}$ & 1 & $1,96^{*}$ & 1 & $1,53^{*}$ & 1 & $0,59^{*}$ \\
\hline 3 & $-1,51^{*}$ & 3 & $-0,86^{*}$ & 2 & $0,85^{*}$ & 2 & $0,80^{*}$ & 2 & $1,31^{*}$ & 2 & $0,88^{*}$ & 2 & $-0,56$ \\
\hline 4 & $-1,44^{*}$ & 4 & $-0,80^{*}$ & 4 & 0,64 & 3 & $-0,64$ & 3 & $0,45^{*}$ & 3 & 0,02 & 3 & $-0,92 *$ \\
\hline 5 & $-1,96^{*}$ & 5 & $-1,31^{*}$ & 5 & $-0,45^{*}$ & 5 & $-5,16^{*}$ & 4 & $0,52^{*}$ & 4 & 0,09 & 4 & $-0,85^{*}$ \\
\hline 6 & $-1,53^{*}$ & 6 & $-0,88^{*}$ & 6 & $-0,02$ & 6 & $-0,09$ & 6 & $0,43^{*}$ & 5 & $-0,43 *$ & 5 & $-1,37^{*}$ \\
\hline 7 & $-0,59^{*}$ & 7 & 0,56 & 7 & $0,92^{*}$ & 7 & $0,85^{*}$ & 7 & $1,37^{*}$ & 7 & $0,94 *$ & 6 & $-0,94 *$ \\
\hline
\end{tabular}

Legenda: *valores com diferenças estatisticamente significativas.

Fonte: Autoras (2013)|.

Analisando os dados, pode ser observado que o Fator 1 (controle da emoção e concentração) apresentou diferenças significativas em relação aos demais Fatores (2, 3, $4,5,6,7)$, sendo o fator que apresentou menor frequência $(7,46)$. Este resultado pode estar relacionado ao fato de ser a EaD um novo contexto de formação, que inclui a necessidade de uma postura diferenciada dos participantes, distinguindo-se das exigidas em outros espaços e modalidades de ensino, podendo assim, desencadear sentimentos de ansiedade e falta de concentração entre os alunos, frente a essa nova modalidade de formação. Ou ainda, como afirmou Paul (1990 apud BELLONI, 2008, p.45), a clientela do curso de $\mathrm{EaD}$ frequentemente experenciou situações educacionais negativas e assim, muitos se acham despreparados, tem problemas de motivação e tendem a se culpar pelos insucessos. Desta forma, afirma o autor que um dos desafios das instituições de EaD seria mais de ordem sócio afetiva, indicando a necessidade de minimizar e até romper sentimentos de ansiedade e de desmotivação.

Estabelecendo comparações entre a média do Fator $2(8,10)$ - busca de ajuda interpessoal, com as médias dos demais fatores da Escala, verifica-se que o Fator 2 apresentou diferenças significativas em relação aos Fatores 3,4,5 e 6, demonstrando 
desta forma, que as estratégias de aprendizagem relacionadas ao Fator 2 foram utilizadas com menor frequência em relação aos demais Fatores, com exceção das estratégias relativas aos fatores 1 (controle da emoção e concentração) e 7 (monitoramento da compreensão), uma vez que não apresentaram diferenças significativas em relação aos dois fatores. Destaca-se neste aspecto a necessidade de estimular os alunos a buscarem a ajuda dos professores, tutores e demais colegas de forma a dirimir eventuais dúvidas sobre o conteúdo estudado.

As comparações realizadas entre o Fator $3(8,97)$ - repetição e organização e os demais fatores indicaram diferenças significativas relacionadas aos Fatores 1, 2 e 7, sendo as estratégias relativas a estes fatores, utilizadas com menor frequência e entre as estratégias do Fator 5, sendo as estratégias de Elaboração (Fator 5) utilizadas com maior frequência em relação as estratégias de Repetição e Organização (Fator 3).

Com relação à média obtida no Fator $4(8,90)$, observou-se que as estratégias de motivação que compõem este Fator são utilizadas com maior frequência quando comparadas aos Fatores 1, 2 e 7. Em relação ao Fator 4, verificou-se que as estratégias deste fator são utilizadas com menos frequência do que as estratégias do Fator 5.

Ao analisar a média do Fator $5(9,42)$, verificou-se diferenças significativas em relação aos fatores $1,2,3,4,6$, sendo estas estratégias utilizadas com menor frequência, quando comparadas ao Fator 5. Pesquisas anteriores como as de Zerbini e Abbad (2008) e Abbad, Corrêa e Meneses (2010) converge com o estudo atual, quando identificaram que as estratégias de Elaboração são utilizadas com mais frequência quando comparadas aos outros fatores da Escala.

Com relação à média do Fator $6(8,99)$ - busca de ajuda ao material didático, foi possível observar que as estratégias de aprendizagem referentes a esse fator, são utilizadas com maior frequência quando comparadas aos Fatores 1, 2, 3, 4, 7. Dentre os Fatores, o que apresentou maior frequência quanto ao uso das estratégias de aprendizagem quando comparado ao Fator 6 foi o Fator 5 (elaboração).

Por fim, quando realizada a comparação do Fator $7(8,05)$ - monitoramento da compreensão, as análises indicaram que as estratégias relacionadas ao Fator 7 são usadas com maior frequência apenas quando comparadas ao Fator 1 e as estratégias de aprendizagem relacionadas aos Fatores 2,3,4,5 e 6 são utilizadas com maior frequência, quando comparadas ao Fator 7.

Tomados em conjunto, os dados evidenciam que as estratégias autorregulatórias de controle da ansiedade e concentração, embora considerada como de alta frequência, foi a estratégia que obteve a menor média. Estas estratégias se referem às emoções diante das possíveis dificuldades a serem experenciadas no decorrer do curso e expectativas em relação aos resultados esperados.

A estratégia de busca de ajuda interpessoal, principalmente ao auxílio de outras pessoas para tirar as dúvidas com colegas e tutores, seja por meio dos chats, lista de discussão, e-mails ou presencias apresentou uma baixa frequência também em relação às demais, assim como a estratégia de monitoramento da compreensão, que envolvem estratégias autorregulatórias de avaliação do próprio processo de aprendizagem e modificação do comportamento, como testar a compreensão de conteúdos por meio de elaboração de perguntas, revisão da matéria para verificação do domínio do conteúdo. Conforme apontado por Boruchovitch (1999), se o aluno não souber como se apropriar, monitorar e regular seu aprendizado, poderá não ter um bom desempenho. Fato este que pode estar relacionado a obtenção de uma menor frequência no uso de estratégias de controle da ansiedade e concentração dos alunos pesquisados, o que merece atenção por parte dos gestores, professores e tutores do curso, havendo necessidade de que as estratégias cognitivas e metacognitivas estejam interligadas. 
Vale ressaltar que as estratégias cognitivas de reflexão do conteúdo (elaboração), que implicam na relação e associação dos novos conteúdos a conhecimentos anteriores, experiências e situações cotidianas foram a utilizadas com maior frequência pelos participantes, o que pode, supostamente estar relacionada ao fato de que todos os alunos pesquisados estavam cursando pedagogia e, portanto, trabalharam conteúdos relativos ao processo ensino-aprendizagem e detinham certa experiência profissional na educação infantil e/ou no ensino fundamental.

\section{Considerações Finais}

As análises realizadas no presente trabalho permitiram identificar o uso das estratégias de aprendizagem cognitivas e metacognitivas utilizadas pelos alunos matriculados no curso de Pedagogia ofertado a distância pela instituição pesquisada e, a partir dessas análises, suscitaram possibilidades de intervenções pedagógicas no contexto da $\mathrm{EaD}$, ao considerar as importantes implicações educacionais relacionadas ao processo ensino-aprendizagem dos alunos neste contexto, em especial, as estratégias de controle da emoção e de monitoramento da compreensão no processo ensinoaprendizagem na EaD.

\section{Agradecimentos}

À Fundação Araucária/PROPPG/UEL pelo apoio à participação em eventos.

\section{Referências}

ABBAD, G.S. et al. Medidas de avaliação em treinamento, desenvolvimento e educação. Rio de Janeiro: Artmed, 2012.

ABBAD, G. S.; CORRÊA, V. P.; MENESES, P.P.M. Avaliação de treinamentos da distância: relações entre estratégias de aprendizagem e satisfação com o treinamento.

Revista de Administração Mackenzie. São Paulo, v.11, n.2. p.43-67, 2010. Disponível em: $<$ http://www.scielo.br/scielo.php?pid=S1678-

69712010000200003\&script=sci_arttext> Acesso em: 04 abr. 2013.

ABED. Associação Brasileira de Educação a Distância. Censo EAD.BR 2011: relatório analítico da aprendizagem a distância no Brasil, 2012. Disponível em:

$<$ http://www.abed.org.br/censoead/CensoEaDbr0809_portugues.pdf $>$. Acesso em: 07 mar. 2013.

BELLONI, M.L. Educação a distância. 5.ed. São Paulo: Autores Associados, 2008.

BOHADANA, E.; VALLE, L. O quem da educação a distância. Revista Brasileira de Educação, v.14, n.42, 2009. Disponível em:

$<$ http://www.scielo.br/pdf/rbedu/v14n42/v14n42a11.pdf >. Acesso em: 07 mar. 2013.

BORGES, V.M.C; JUNIOR, T.C.B. Perfis de uso de estratégias de aprendizagem de alunos em ambiente virtual. Horizonte de Linguagem Aplicada, Brasília, v. 11, n.1, p. 59-80, 2012. Disponível em:

$<$ http://seer.bce.unb.br/index.php/horizontesla/article/view/6121> Acesso em: 29 out 2013. 
BORUCHOVITCH, E. Estratégias de aprendizagem e desempenho escolar: considerações para a prática educacional. Psicologia Reflexão e Crítica. Porto Alegre, v.12, n. 2, 1999. Disponível em:

$<$ http://www.scielo.br/scielo.php?script=sci_arttext\&pid=S010279721999000200008\&1 ng=pt\&nrm=iso $>$ Acesso em: 14 abril 2013.

BUSNELLO, F.B.; JOU, G.I.; SPERB, T.M. Desenvolvimento de habilidades metacognitivas: capacitação de professores de ensino fundamental. Psicologia:

Reflexão e Crítica. Porto Alegre, v.25, n.2, p.311-319, 2012. Disponível em: $<$ http://www.scielo.br/scielo.php?pid=S0102-79722012000200013\&script=sci_arttext $>$. Acesso em: 17 ABR. 2013.

COOL, C.; MONEREO, C. Psicologia da educação virtual. Porto Alegre: Artmed, 2010 .

CORSO, H.V. et al. Metacognição e funções executivas: relações entre os conceitos e implicações para a aprendizagem. Psicologia: teoria e pesquisa. Brasília, v.29, n.1, p.21-29, 2013. Disponível em: $<$ https://revistaptp.unb.br/index.php/ptp/article/view/359>. Acesso em: 18 jun.2013.

COSTA, E.R; BORUCHOVITCH, E. As estratégias de aprendizagem e a produção de textos narrativos. Psicologia: Reflexão e Crítica. Porto Alegre, v. 22, n.2,p. 173-180, 2009. Disponível em: <http://www.scielo.br/scielo.php?pid=S0102-

$79722009000200002 \&$ script=sci_arttext $>$. Acesso em: 15 abril 2013.

FARIA, E. V. Tutor na educação à distância: a construção de conhecimentos pela interação nos ambientes midiáticos no contexto da educação libertadora. 2010. Revista Scientia FAER. v. 2, n. 2, p. 28-37, 2010. Disponível em: $<$ http://www.faer.edu.br/revistafaer/artigos/edicao2/elisio.pdf. $>$ Acesso em: 09 mar. 2013.

JOLY, M.C.R.A. Escala de estratégia de leitura-formato ensino fundamental: pesquisa em desenvolvimento. Itatiba: Universidade de São Francisco, 2004.

LEME, E.M. Estilos e estratégias de aprendizagem: estudo das relações entre os construtos. Dissertação (Mestrado em Psicologia) - Universidade São Francisco, Itatiba, 2010. Disponível em:

$<$ http://www.usf.edu.br/itatiba/mestrado/psicologia/uploadAddress/Erika_Monqueiro_L eme\%5B13761\%5D.pdf>. Acesso em: 15 abr. 2013.

LINS, M.R.C; ARAUJO, M.R; MINERVINO, C.A.S.M. Estratégias de aprendizagem empregadas por estudantes do ensino fundamental. Revista Semestral da Associação Brasileira de Psicologia Escolar e Educacional, São Paulo, v. 15, n.1, p.63-70, 2011. Disponível em: <http://www.scielo.br/pdf/pee/v15n1/07.pdf>. Acesso em: 17 jun. 2013.

TANNOUS, K.; ROPOLI, E. Análise dos aspectos motivacionais relacionados à evasão e à aprovação em um curso de extensão, 05/2005. ABED. Associação Brasileira de Educação a Distância. Disponível em:

$<$ http://www.abed.org.br/congresso2005/por/pdf/152tcc5.pdf $>$. Acesso em 15 fev. 2013. 
ZERBINI, T; ABBAD, G. Impacto de treinamento no trabalho via internet. RAE Eletrônica. Brasília, v. 4, n.2, 2005. Disponível em:

$<$ http://rae.fgv.br/sites/rae.fgv.br/files/artigos/10.1590_S1676-5648200500020002.pdf> Acesso em: 12 abr. 2013.

ZERBINI, T. ABBAD, G. Estratégias de aprendizagem em curso a distância: validação de uma escala. Psico USF. São Paulo, v. 13, n. 2, p.177-187, 2008. Disponível em: $<$ http://www.scielo.br/scielo.php?pid=S1413-82712008000200005\&script=sci_arttext.> Acesso em: 29 out. 2013.

ZERBINI, T; PILATI, R. Medidas de insumo: perfil cognitivo-comportamental da clientela de ações de TDeE. In: ABBAD, G.S. et al. Avaliação em treinamento, desenvolvimento e educação. Porto Alegre: Artmed, 2012. p. 226-243. 